\title{
Abstandsgewirke und andere Abstandsgewebe
}

\author{
U. Wollina' \\ Marianne Heide ${ }^{2}$ \\ M. Swerew ${ }^{3}$ \\ M. Billia ${ }^{4}$ \\ U. Möhring ${ }^{2}$
}

\section{Spacer Fabrics and Related Textile Solutions}

\section{Zusammenfassung}

Abstandsgewirke sind spezielle Textilien mit Produkteigenschaften wie Druckentlastung, Regulation von Wärme- und Flüssigkeitstransport. Textilphysiologische Untersuchungen verweisen auf günstige Produkteigenschaften, die einen Einsatz in der Medizin favorisieren. Die Anwendung von Abstandsgewirken zur Vermeidung chronischer Wunden steht erst am Anfang. Doch bereits jetzt lassen sich typische Einsatzgebiete erkennen, wie die primäre Prävention des Dekubitus im Operationssaal, die sekundäre Dekubitusprävention bei Rollstuhlfahrern oder die Vorbeugung von Plantarulzera bei Patienten mit dem diabetischen Fuß-Syndrom. Bandagen auf der Basis von Abstandsgewirken scheinen hilfreich bei der Ödembehandlung im Rahmen der chronisch-venösen Insuffizienz oder des Lymphödems. Verwandte Lösungen sind auf der Basis von Gestricken entwickelt und zur lokalen Behandlung chronischer Wunden bereits in die Praxis eingeführt worden. Weitere Entwicklungen z.B. im Bereich Tissue Engineering sind auf diesem Sektor zu erwarten.

\section{Abstract}

Spacer fabrics are special textiles that offer designed qualities for pressure relief, regulation of heat and moisture. Textile physiological investigations demonstrate favourable qualities for their use in the medical field. The application of spacer fabrics for prevention of chronic wounds is just at the beginning. However, typical fields of application are primary prevention of pressure sores in the operation room, secondary prevention of pressure sores for wheel chair drivers and the prevention of plantar ulcers in patients with diabetic foot syndrome. The use of spacer fabric based bandages seems to be useful in edema treatment in case of chronic venous insufficiency and chronic lymphatic insufficiency. Comparable solutions have been developed on the basis of embroidery. Products are on the market now for topical treatment of chronic wounds. Further developments, e.g. for tissue engineering, may be available in the future.

\section{Einleitung}

Es besteht kein Zweifel, dass aufgrund der Altersstruktur der Bevölkerung in den Industriestaaten und der epidemiologischen Situation bei Stoffwechsel- und Ernährungskrankheiten mit einer Zunahme chronischer Wundpatienten zu rechnen ist.
Schätzungsweise $60 \%$ bis $80 \%$ aller chronischen Wundpatienten leiden an venösen und gemischten venös-arteriellen Ulzera der unteren Extremität. Neuropathische Ulzera sind vergleichsweise seltener, verursachen jedoch z.T. hohe Folgekosten und bergen auch ein hohes Amputationsrisiko.

Institutsangaben

${ }^{1}$ Hautklinik, Krankenhaus Dresden-Friedrichstadt, Städtisches Klinikum, Dresden

${ }^{2}$ Textilforschungsinstitut Thüringen - Vogtland e.V., Greiz

${ }^{3}$ Bekleidungsphysiologisches Institut Hohenstein e. V., Bönnigheim

${ }^{4}$ TISSUPOR AG, St. Gallen

Korrespondenzadresse

Prof. Dr. U. Wollina · Hautklinik, Krankenhaus Dresden-Friedrichstadt · Städtisches Klinikum .

Friedrichstraße 41 · 01067 Dresden · E-mail: Wollina-Uw@khdf.de

Bibliografie

Akt Dermatol 2004; 30: 8-10 @ Georg Thieme Verlag Stuttgart · New York · ISSN 0340-2541.

DOI $10.1055 / \mathrm{s}-2004-814273$ 
Dekubitalgeschwüre sind ein Problem im Qualitätsmanagement operativer Disziplinen, der Neurologie und Geriatrie. Prävention und Behandlung sind mit einem hohen Personalaufwand verknüpft. Alle chronischen Wunden reduzieren die Lebensqualität der Patienten, verursachen soziale und medizinische Kosten und stellen eine hohe Belastung des Gesundheitshaushaltes dar $[1-4]$.

Dabei sind die Hauptkosten der medizinischen Behandlung bei den Diagnosegruppen durchaus unterschiedlich verteilt. So wird bei venösen Ulzera der Hauptanteil der Kosten durch ambulante Versorgung, beim diabetischen Fuß-Syndrom jedoch durch stationäre, meist operative Behandlung bedingt [5-7].

Zur Verbesserung dieser Situation sind Neuentwicklungen auf dem Gebiet der Wundversorgung, Primär- und Sekundärprävention sowie strukturelle und organisatorische Veränderungen in den letzten Jahren eingeführt worden. Textile Lösungen sind vergleichsweise unterschätzte Ansätze, obgleich deren Potenzial in der Praxis ganz erheblich sein kann [8-12]. Eine derartige textile Lösung stellen Abstandsgewebe dar, die im Folgenden besprochen werden sollen [13].

\section{Abstandsgewirke}

Abstandsgewirke stellen eine Kombination von Polfäden mit zwei textilen Flächen dar. Durch geeignetes Design der Komponenten kann ein funktionelles Textil für verschiedene Anwendungen entwickelt werden. Die Herstellung erfolgt mit einer Raschelmaschine mit 6 Grundlegebarren, Zungennadel-, Abschlagund Stechkammbarren. Der Abstand der Nadelbarren ist variabel. Auf diese Weise kann die erforderliche Dicke des Textils und das Luftvolumen zwischen den beiden textilen Außenflächen bestimmt werden (Abb.1) [14]. Durch Auswahl der Polfäden werden Steifigkeit, Elastizität, Druckverteilung, Feuchteund Wärmeleitvermögen beeinflusst. Monofile, kapilläre, multifile Fasern sind möglich. Durch chemische Modifikationen an der Oberfläche oder Kombination von Fasern wird die Palette techni-

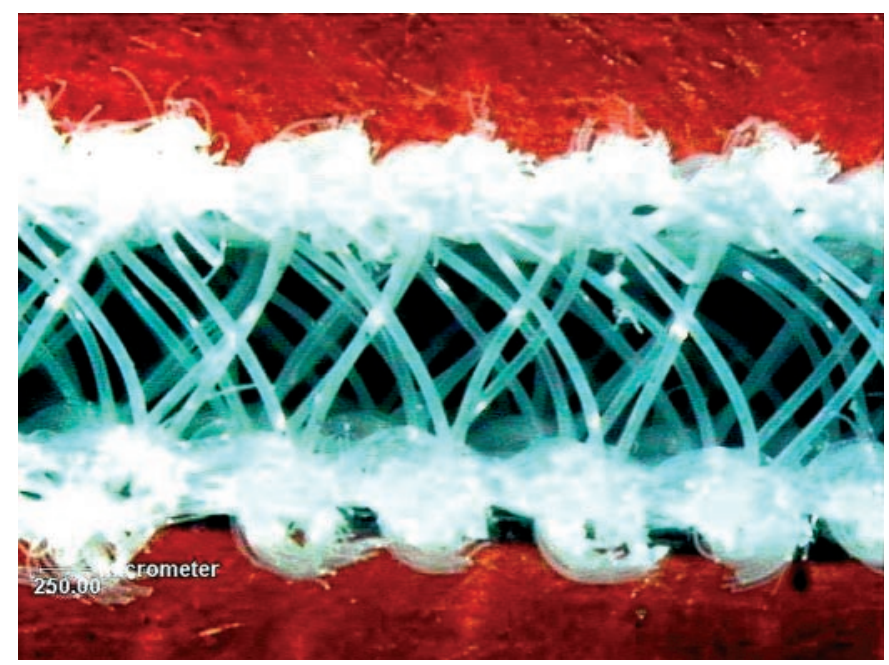

Abb. 1 Querschnitt durch ein Abstandsgewirke. Man erkennt sehr schön die Anordnung der Polfäden, die an eine Brückenkonstruktion erinnert. scher Möglichkeiten vergrößert. So sind beispielsweise Flüssigkeitsaufnahmen von den textilen Oberflächen in weniger als 3 Sekunden zu realisieren. Der Sorptions-Index erreicht Werte von 0,1 Sekunden. Dampfdiffusionswerte erreichen die Größenordnung, die für körperanliegende Wäsche nach DIN EN 31092 empfohlen werden [15]. Ein gerichteter Flüssigkeitstransport wird beispielsweise durch Zellulosefasern oder Kombinationen von synthetischen Fasern möglich [16]. Das Wärmeisolationsverhalten ist günstiger als für etablierte OP-Tischauflagen auf Gelbasis [15]. Damit verringert sich das Risiko der Auskühlung von Patienten im OP und somit auch das Risiko der Dekubitusentstehung. Dies gilt im Prinzip auch für Bettaustattungen mit einem solchen Textil. Hierbei ist die Inkontinenz ein Risikofaktor der Dekubitusentstehung. Gerichteter Flüssigkeitstransport weg vom Patienten hilft in der Dekubitusprävention [17].

Druckentlastung wird durch Veränderung des Polfadenwinkels, der Polfadendichte und des Polfadenmaterials erzielt. Im Rahmen von Dummy-Experimenten konnte eine erhebliche Reduktion von Druckspitzen z.B. im Beckengürtelbereich erzielt werden. Minderungen des mittleren Auflagedruckes von bis zu 25\% konnten durch leichte Abstandsgewirke erreicht werden. In einer Anwendungsbeobachtung über 12 Monate wurden im eigenen OP keine Auskühlung oder Dekubitusentwicklung festgestellt. Von Vorteil war auch die geringe Flächendichte im Vergleich zu herkömmlichen Gelmatten. Auf diese Weise ist ein besseres Handling für OP-Pflegekräfte möglich [vgl. 17].

\section{Gesticke als Spacer}

Sticktechniken erlauben es, dreidimensionale textile Strukturen mit strukturierter Oberfläche zu erzeugen. Diese technologischen Lösungen sind auch für schlauchartige Gebilde z.B. in der Gefäßprothetik denkbar [18]. Im Rahmen der Wundbehandlung sind mechanische Stimulation und Flüssigkeitstransport von größerer Bedeutung. Auf der Grundlage der Stickereitechnik wurde ein funktionelles Wundpad entwickelt (Tissupor ${ }^{\circledR}$, Abb. 2a). Der Wunde zugewandt ist eine gestickte Grundstruktur aus PET/Polyamid. Ein daran anschließendes Abstandsgewirke aus PES übernimmt Scherkräfte, überträgt Kompression und absorbiert Wundexsudat in einer Viskose-Vliesschicht. Den Abschluss bildet ein dichtes Gewebe aus PES. Es garantiert mechanischen Schutz und kontrolliert zudem den Feuchtigkeits- und Gastransport. Aufgrund der wundseitigen Porengröße von 10 - $300 \mu \mathrm{m}$ ist ein Einwachsen von Granulationsgewebe möglich (Abb. 2 b). Über lokale mechanische Stimulation wird die Granulation und Neoangiogenese gefördert [19]. In-vitro-Untersuchungen mit 3T3-Fibroblasten in verschiedenen ZytotoxizitätsAssays konnten keinerlei zytotoxische Wirkungen nachweisen. Eine Studie im Schweizer Paraplegie-Zentrum Nottwil an $35 \mathrm{~Pa}-$ tienten mit Dekubitus zeigte eine Stimulation der Wundheilung bei guter Verträglichkeit [20]. Von Vorteil ist der seltenere Verbandwechsel im Vergleich zur konventionellen Behandlung. Eigene, unkontrollierte Beobachtungen bei chronischen Wunden sprechen für Einsatzmöglichkeiten bei tieferen Ulzera auf der Basis von venösen oder gemischt venös-arteriellen Gefäßleiden, bei sekundär heilenden OP-Wunden, beim diabetischen FußSyndrom und Dekubitus, Phlegmone (nach chirurgischer Spaltung) und anderen Weichteildefekten [19]. 

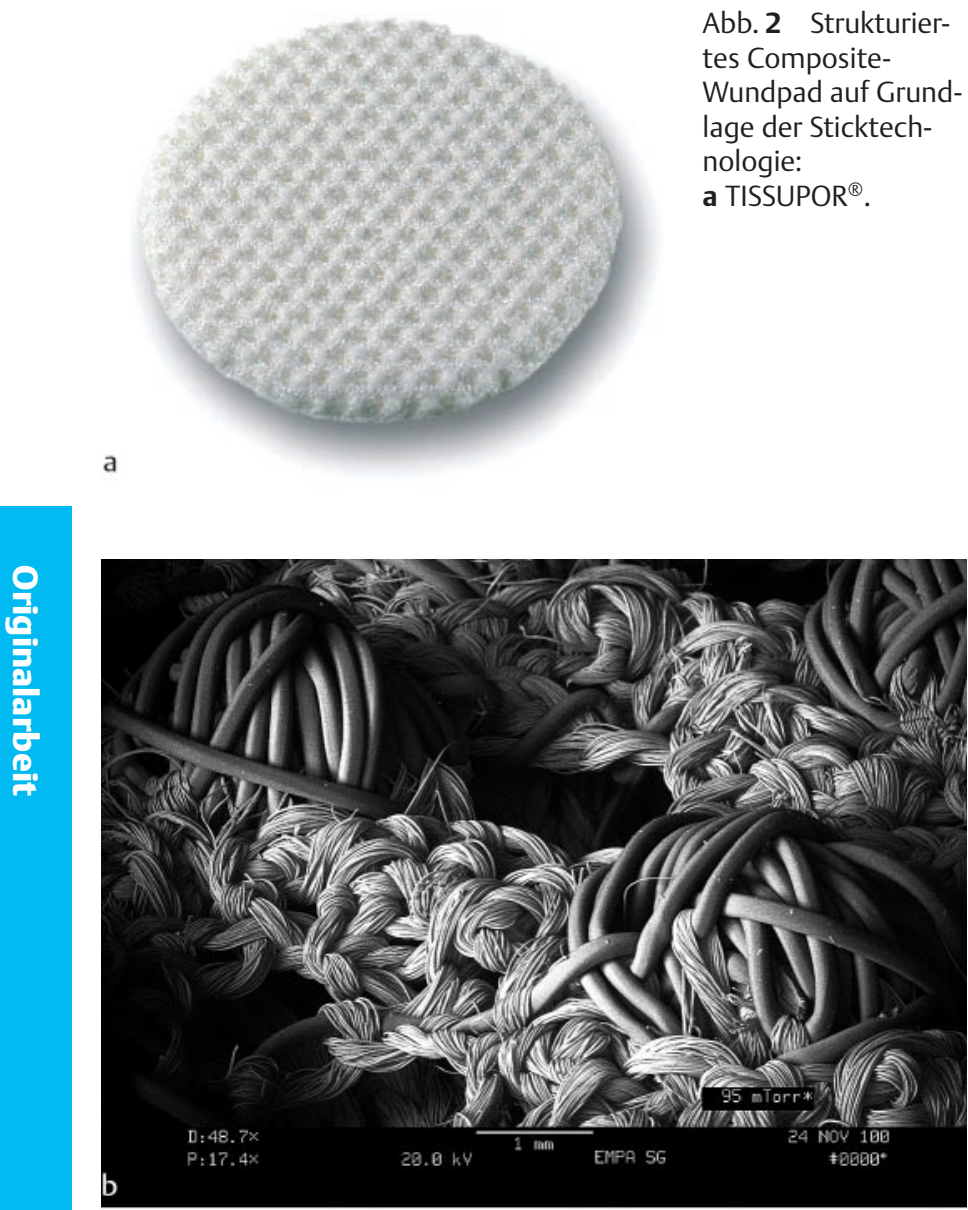

b Scanning-Elektronenmikroskopie der strukturierten, verschiedenporigen Oberfläche zur Stimulation der Neoangiogenese.

Ausblick

Textile Lösungen für Wundprävention und -behandlung sind Erfolg versprechend, wenn diese in ein Wundmanagement eingebettet werden. In der Zukunft sind Designerlösungen für spezielle Problemfelder zu erwarten. Textile Technologien sind auch für biodegradable Wundauflagen oder Scaffolds im Rahmen des Tissue Engineerings zu erwarten. Dabei könnte der Einsatz natürlicher Fasermaterialien von größerem Interesse sein [vgl. 17, 21].
Literatur

${ }^{1}$ Valencia IC, Falabella A, Kirsner RS, Eaglstein WH. Chronic venous insufficiency and venous leg ulceration. J Am Acad Dermatol 2001; 44: $401-421$

${ }^{2}$ Apelqvist J. Wound healing in diabetes. Outcome and costs. Clin Podiatr Med Surg 1998; 15: 21 - 39

${ }^{3}$ Wollina U. Therapie chronischer Wunden - Stiefkind der modernen Medizin? Akt Dermatol 2000; 26 (Suppl 1): S20-S27

${ }^{4}$ Schmidt S, Wollina U, Looks A, Elsner P, Strauss B. Quality of life and strategies of coping with disease in patients with chronic leg ulcers. Dermatol Psychosomatics 2000; 1: 27-34

${ }^{5}$ Levy E, Levy P. Management of venous leg ulcer by French physicians, diversity and related costs: a prospective medicoeconomic observation study. J Mal Vasc 2001; 26: 39-44

${ }^{6}$ Harrington C, Zagari MJ, Corea J, Klitenic J. A cost analysis of diabetic lower-extremity ulcers. Diabetes Care 2000; 23: 1333-1338

${ }^{7}$ Tenvall GR, Apelqvist J, Eneroth M. Costs of deep foot infections in patients with diabetes mellitus. Pharmacoeconomics 2000; 18: $225-238$

${ }^{8}$ Apelqvist J, Larsson J. What is the most effective way to reduce incidence of amputation in the diabetic foot? Diabetes Metab Res Rev 2000; 16 (Suppl 1): S75-S83

${ }^{9}$ Bergemann R, Lauterbach KW, Vanscheidt W, Neander KD, Engts R. Economic evaluation of the treatment of chronic wounds: hydroactive wound dressings in combination with enzymatic ointment versus gauze dressings in patients with pressure ulcer and venous leg ulcer in Germany. Pharmacoeconomics 1999; 16: 367-377

${ }^{10}$ Capillas Perez R, Cabre Aguilar V, Gil Colome AM, Gaitano Garcia A, Torra i Bou JE. Comparision of the effectiveness and costs of treatment with humid environment as compared to traditional cure. Clinical trial on primary care patients with venous leg ulcers and pressure sores. Rev Enferm 2000; 23: 17-24

${ }^{11}$ Morell CJ, Walters SJ, Dixon S, Collins KA, Brereton LML, Peters J, Brooker CGD. Cost effectiveness of community leg ulcer clinics: randomised controlled trial. Br Med J 1989; 316: 1487-1491

12 Kantor J, Margolis DJ. Treatment options for diabetic neuropathic foot ulcers: a cost-effectiveness analysis. Dermatol Surg 2001; 27: $347-351$

${ }^{13}$ Wollina U, Heide M, Müller-Litz W. Stellen Medizin und Gesundheitswesen neue Anforderungen an die Textilqualität? Melliand Textilber 1998; 79: $552-553$

${ }^{14}$ Heide M. Dreidimensional gewirkte Textilien in der Medizin. Maschen-Industrie 1999; 6: 14-17

${ }^{15}$ Möhring U, Mecheels S, Stoll M (eds). Entwicklung funktioneller Abstandsgewirke mit definiertem druckelastischen Verhalten und Thermoregulation für den medizinischen Bereich. AiF-Verbundvorhaben Nr. 1 ZGB/1, /2, und /3. Abschlussbericht 2001

${ }^{16}$ Mayrowitz HN, Sims N. Biophysical effects of water and synthetic urine on skin. Adv Skin Wound Care 2001; 14: 302 - 308

${ }^{17}$ Wollina U, Heide M, Uhlemann C, Neupert G, Obenauf D. Spacer fabrics in der Dermatologie. Eine Übersicht. Z Wundheilung - J Wound Healing 2000; 5(No 13): 7-11

${ }^{18}$ Karamuk E, Mayer J. Embroidery technology for medical textiles and tissue engineering. Techn Textiles International 2000; 9: 9-13

${ }^{19}$ Karamuk E, Mayer J, Selm B, Bischoff B, Ferrario R, Heller M, Billia M, Seidel R, Wanner E, Moser R. Development of a structured wound dressing based on a textile composite functionalised by embroidery technology. KTI Projekt No. 511. September 2001

${ }^{20}$ Wanner M, Karamuk E, Billia M, Ferrario R, Wagner B, Moser R, Mayer J. Preliminary results of a new type of wound treatment. Swiss Paraplegic Centre Nottwil

21 Vollrath F, Bart P, Basedow A, Engstrom W, List H. Local tolerance to spider silks and protein polymers in vivo. In Vivo 2002; 16: 229-234 